\title{
Gastric schwannoma exhibiting increased fluorodeoxyglucose uptake
}

\author{
Daisuke Komatsu ${ }^{1}$, Naohiko Koide ${ }^{1}$, Risako Hiraga ${ }^{1}$, Naoyuki Furuya ${ }^{2}$, Taiji Akamatsu ${ }^{3}$, Takeshi Uehara $^{4}$, \\ and SHinichi Miyagawa ${ }^{1}$ \\ ${ }^{1}$ Department of Surgery, Shinshu University School of Medicine, 3-1-1 Asahi, Matsumoto, Nagano 390-8621, Japan \\ ${ }^{2}$ Department of Internal Medicine, Hotaka Hospital, Azumino, Nagano, Japan \\ ${ }^{3}$ Department of Endoscopy, Shinshu University School of Medicine, Matsumoto, Nagano, Japan \\ ${ }^{4}$ Department of Laboratory Medicine, Shinshu University School of Medicine, Matsumoto, Nagano, Japan
}

\begin{abstract}
This is the first case of gastric schwannoma that exhibited increased accumulation of [(18)F] fluorodeoxyglucose (FDG) on positron emission tomography (PET) imaging. The patient was a 60-year-old woman in whom esophagogastroduodenoscopy showed a submucosal tumor, about $25 \mathrm{~mm}$ in size, in the upper body of the stomach, with ulceration at the top of the tumor. Endoscopic ultrasonography revealed a well-defined hypoechoic mass located in the proper muscle layer of the stomach. The specimen taken from the tumor showed only inflammatory degenerative tissue. Abdominal computed tomography revealed a tumor in the upper body of the stomach. FDG-PET showed FDG uptake (standardized uptake value [SUV] max 5.8) coincident with the tumor. Hence, the tumor was diagnosed initially as a gastrointestinal stromal tumor of the stomach. Laparoscopic partial gastrectomy was performed. Pathological examination showed that the tumor consisted of spindle cells with large nuclei, and mitosis was absent. The Ki-67 labeling index of the tumor cells was $4 \%$. Immunohistochemically, the tumor cells showed a positive reaction for $\mathrm{S}-\mathbf{1 0 0}$ protein, whereas they were negative for KIT, CD 34, and alpha-smooth muscle actin protein. The tumor was diagnosed as a benign gastric schwannoma. Gastric schwannoma should be included in the differential diagnosis of submucosal tumors of the stomach with FDG uptake.
\end{abstract}

Key words Schwannoma - Stomach - Fluorodeoxyglucose • Positron emission tomography

\section{Introduction}

Most gastrointestinal (GI) schwannomas are benign mesenchymal tumors composed of schwann cells, which physiologically wrap around the axons of peripheral

Offprint requests to: $\mathrm{N}$. Koide

Received: June 23, 2009 / Accepted: October 5, 2009 nerves [1]. According to a recent classification, about $80 \%$ of GI mesenchymal tumors are gastrointestinal stromal tumors (GISTs) [2]. GI schwannomas have been reported to represent only $3 \%$ of all GI mesenchymal tumors [3]. GI schwannomas occur predominantly in females in their 50s. [4]. The stomach is the most common site of GI schwannomas, which constitute $0.2 \%$ of all gastric neoplasms [5]. Recently, [(18)F] fluorodeoxyglucose (FDG) positron emission tomography (PET), an evolving diagnostic modality, has been used for tumor detection, staging, therapeutic monitoring, and follow-up evaluations for various malignant tumors $[6,7]$. FDG-PET has been adopted to distinguish benign tumors from malignant mesenchymal tumors, such as GISTs. We report herein the first case of benign gastric schwannoma showing increased FDG uptake on PET.

\section{Report of a case}

A 60-year-old woman received esophagogastroduodenoscopy (EGD) for stomach cancer screening. EGD showed an elevated submucosal tumor with bridging folds in the upper body of the stomach. The submucosal tumor had a central ulcer on its top (Fig. 1A). Endoscopic ultrasonography (EUS) revealed a wellcircumscribed hypoechoic mass, $25 \mathrm{~mm}$ in size, arising from the proper muscle layer of the stomach (Fig. 1B). Histopathologically, biopsy specimens taken from the ulcerative lesion of the submucosal tumor showed degenerative tissues with infiltration of neutrocytes. Abdominal computed tomography (CT) confirmed the presence of this tumor in the upper body of the stomach (Fig. 2). FDG-PET showed high accumulation (standardized uptake value [SUV] max 5.8) coincident with the gastric tumor (Fig. 3). Consequently, GIST of the stomach was the most likely diagnosis. Laparoscopically, the tumor was located in the greater curvature of the stomach, and a wedge resection of the stomach with 

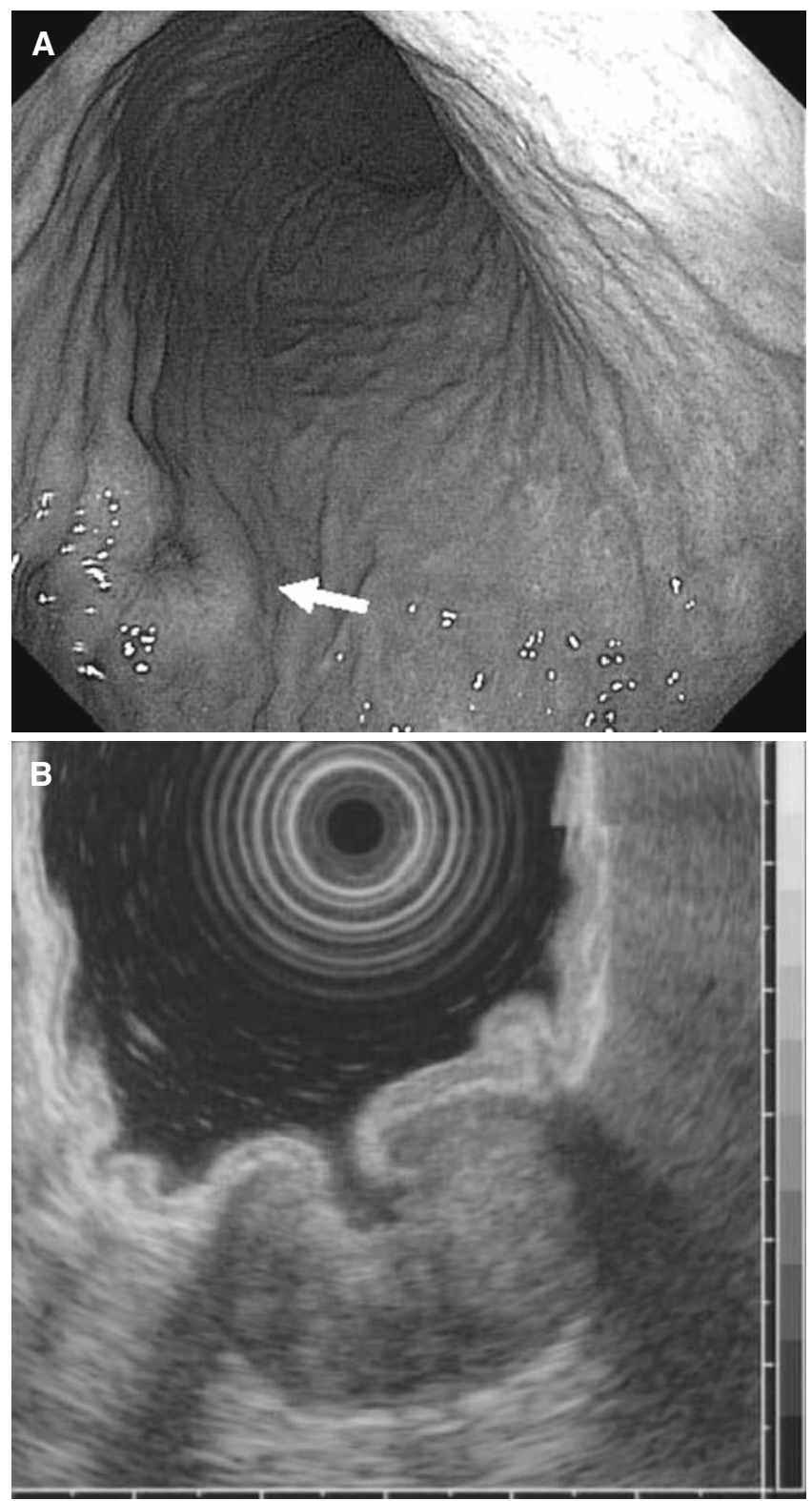

Fig. 1A,B. Endoscopic findings. A The arrow indicates a submucosal tumor with a central ulceration on its top in the anterior wall of the upper body of the stomach. B Endoscopic ultrasonography showed a well-circumscribed hypoechoic mass, $25 \mathrm{~mm}$ in size, arising from the proper muscle layer of the stomach

adjacent lymph nodes was performed. Macroscopically, the removed tumor was well circumscribed and measured $25 \times 25 \times 15 \mathrm{~mm}$, with a 5 -mm ulceration at the center of the tumor. The cut surface of the tumor appeared yellowish-white and solid (Fig. 4). Histopathologically, the tumor was located mainly in the proper muscle layer of the stomach, and consisted of spindle cells with large nuclei and vague nuclear palisading (Fig. 5A). Mitoses, necrosis, and bleeding were absent. The resected lymph nodes showed no metastasis. The Ki-67

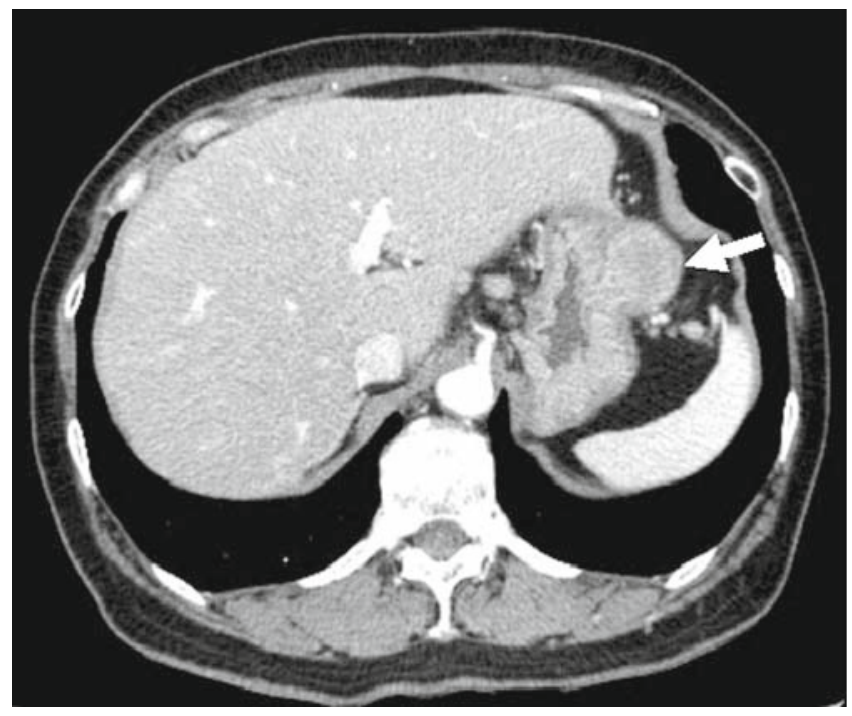

Fig. 2. Computed tomography (CT) findings. Contrastenhanced CT shows a discrete and homogeneous mass in the upper body of the stomach (arrow)

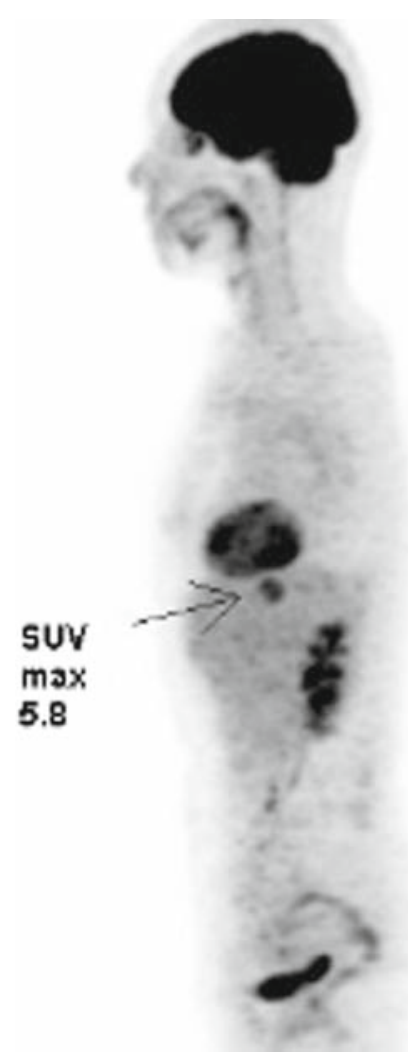

Fig. 3. Positron emission tomography (PET) findings. [(18)F] Fluorodeoxyglucose (FDG)-PET shows high accumulation (standardized uptake value $[S U V] \max 5.8$ ) coincident with the gastric tumor

labeling index of the tumor cells was $4 \%$. Immunohistchemical staining showed that the cytoplasm of the tumor cells was diffusely positive for S-100 protein (Fig. $5 \mathrm{~B}$ ), whereas the tumor cells were negative for KIT, CD 


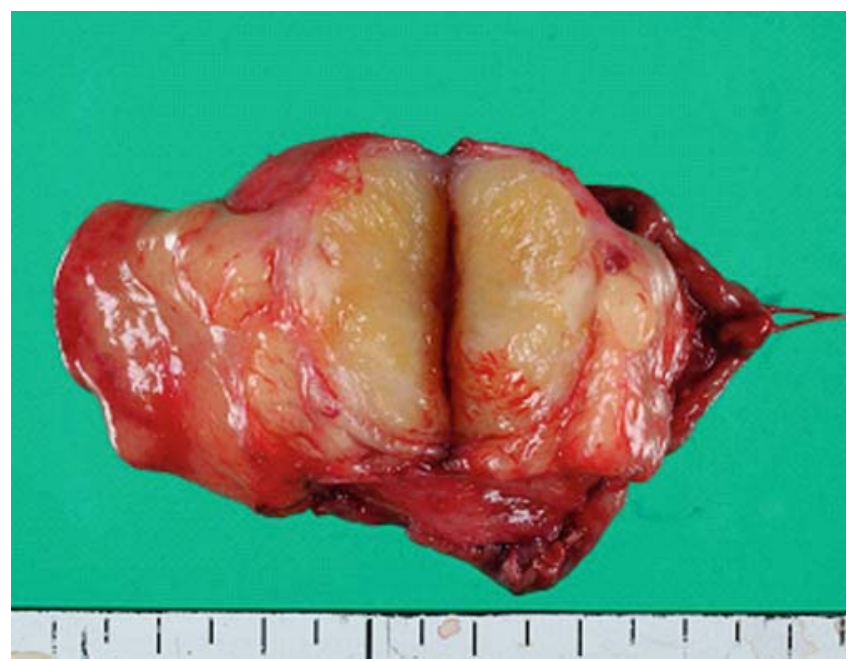

Fig. 4. The gross appearance of the resected specimen. A well-demarcated submucosal tumor is observed in the stomach. The cut surface of the tumor appears solid and yellowish-white

34, and alpha-smooth muscle actin protein. Furthermore, a cuff-like lymphoid aggregate was recognized around the tumor (Fig. 5C). The tumor was diagnosed as a benign gastric schwannoma. Ten months after the surgery, the patient is well without any evidence of recurrent disease.

\section{Discussion}

Schwannomas have a predilection for the head, neck, and flexor surfaces of the limbs, but they can occur anywhere in the body [1]. Although schwannomas can also arise throughout the GI tract, they are most commonly found in the stomach [5]. Patients with gastric schwannoma are usually asymptomatic, and therefore most of these tumors are discovered incidentally [5]. Gastric schwannomas are most commonly intramural $(65 \%)$, but they can be intraluminal or subserosal as well. The tumors arise most commonly from the body of the stomach $(50 \%)$ with fewer developing in the antrum $(32 \%)$ or the fundus (18\%) [8]. Unlike GISTs, gastric schwannomas are benign; malignant transformation is extremely rare [9]. EGD is commonly used as a diagnostic tool for several types of gastric tumors. Endoscopically, gastric schwannomas appeared as elevated submucosal masses [10]. A central ulcer was seen in $25 \%-50 \%$ of gastric schwannomas [10, 11]. Falsenegative results of endoscopic biopsy diagnosis would be encountered, because the normal mucosa frequently overlies gastric schwannomas [12]. As shown in our case and other cases $[13,14]$, endoscopic biopsy revealed only chronic inflammation without any malignant cells.
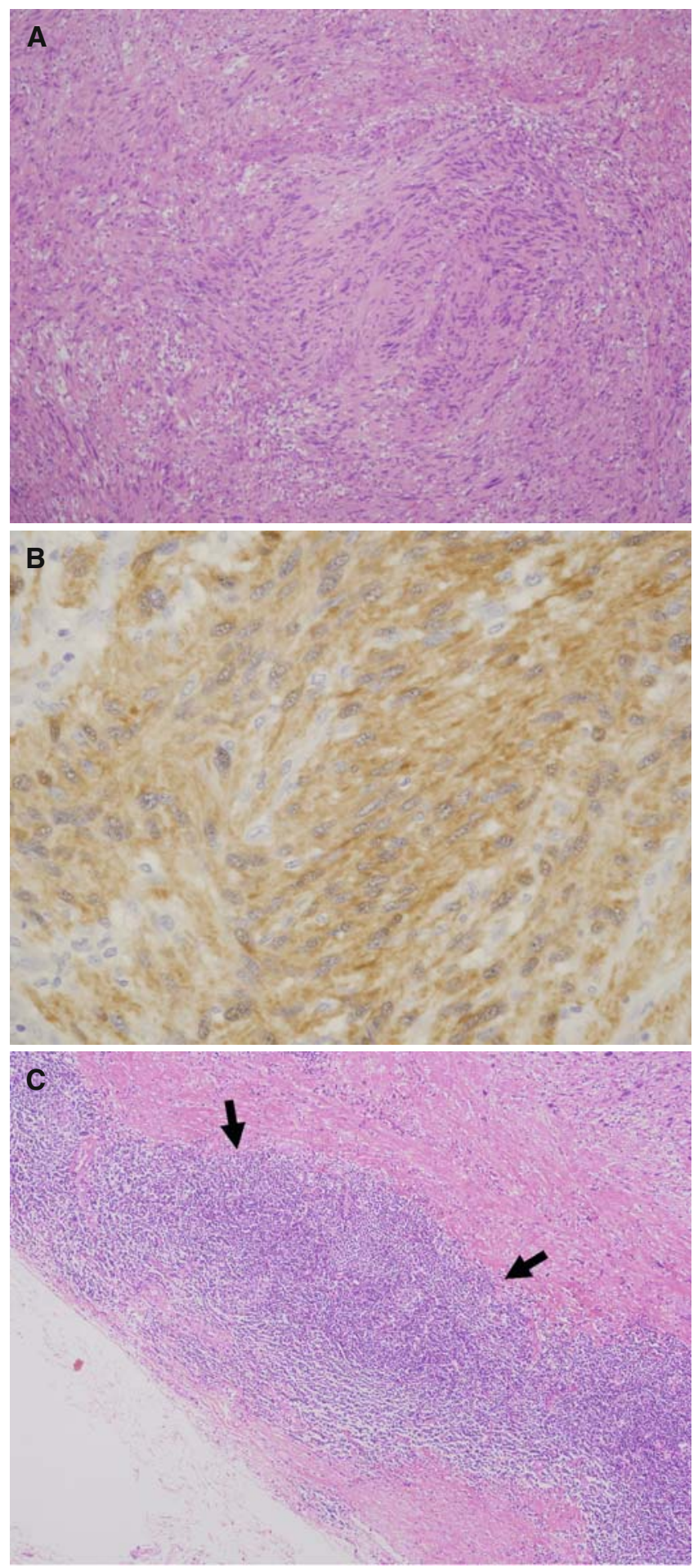

Fig. 5A-C. Histopathological findings. A The tumor consists of spindled cells with large nuclei and vague nuclear palisading. B Immunohistochemical staining. The spindle tumor cells show a positive reaction for S-100 protein. C A cuff-like lymphoid aggregate was recognized around the tumor (arrows). $\mathbf{A}$ and $\mathbf{C ~ H} \& \mathrm{E}, \times 25 ; \mathbf{B} \times 100$ 
CT shows that gastric schwannomas are well-defined, round, mural masses with homogeneous attenuation. Schwannomas may appear exophytic or project intraluminally, and they may exhibit an ulcer crater. Of note, gastric schwannomas typically, as in the present case, show no evidence of necrosis, hemorrhage, or cystic change on CT. In contrast, these CT findings are common in GISTs [13].

The preoperative differential diagnosis of a gastric submucosal tumor includes GIST, leiomyoma or leiomyosarcoma, and schwannoma. Histologically, GIST may especially mimic a schwannoma and consist of spindled cells with nuclear palisading. The definitive diagnosis of GI schwannomas is made by immunohistochemistry. Schwannomas show diffuse cytoplasmic immunoreactivity for S-100 protein. Mitotic figures are rare or absent [3]. Recently, FDG-PET has been accepted as a powerful and noninvasive metabolic imaging modality for evaluating various tumors, including their detection, distinguishing their malignant potential preoperatively, and staging and monitoring therapeutic response [6,7]. Ahmed et al. [15] reported FDG uptake in 22 benign schwannomas of the extremities, and several authors have previously reported schwannomas in the retroperitoneum [16] and in the esophagus [17] with increased FDG uptake. To our best knowledge, there has been no previous report of gastric schwannoma with increased FDG uptake in the Englishlanguage literature.

It has not been clarified why benign schwannomas show increased FDG uptake on PET. The large accumulation of FDG in schwannomas may result the potential ability of schwann cells to transport glucose for axonal repolarization [18]. Additionally, in 97\% of gastric schwannomas, cuff-like lymphoid aggregates were recognized around the tumors [19], as occurred in the present case. Kawabe et al. [20] reported that FDG accumulated in inflammatory tissue involving lymphocyte proliferation in chronic tonsillitis. Although further studies are necessary to elucidate this mechanism.

In conclusion, we have reported the first case of gastric schwannoma with increased FDG uptake. Gastric schwannomas cannot be differentiated from GISTs by the presence of FDG accumulation, so the evaluation of whether the tumors are benign or malignant should be made by histological diagnosis.

\section{References}

1. Enzinger FM, Weiss SW. Benign tumors of peripheral nerves. In: Enzinger FM, Weiss SW, editors. Soft tissue tumors. 2nd ed. St Louis: Mosby; 1988. p. 725-35.
2. Miettinen M, Sarlomo-Rikala M, Lasota J. Gastrointestinal stromal tumors: recent advances in understanding of their biology. Hum Pathol 1999;30:1213-20.

3. Daimaru Y, Kido H, Hashimoto H, Enjoji M. Benign schwannoma of the gastrointestinal tract: a clinicopathologic and immunohistochemical study. Hum Pathol 1988;19:257-64.

4. Kwon MS, Lee SS, Ahn GH. Schwannomas of the gastrointestinal tract: Clinocopathological features of 12 cases including a case of esophageal tumor compared with those of gastrointestinal stromal tumors and leiomyomas of the gastrointestinal tract. Pathol Res Pract 2002;198:605-13.

5. Melvin WS, Wilkinson MG. Gastric schwannoma. Clinical and pathologic considerations. Am Surg 1993;59:293-6.

6. Hustinx R, Benard F, Alavi A. Whole-body FDG-PET imaging in the management of patients with cancer. Semin Nucl Med 2002;32:35-46.

7. Bomanji JB, Costa DC, Ell PJ. Clinical role of positron emission tomography in oncology. Lancet Oncol 2001;2:157-64.

8. Bruneton JN, Drouillard J, Roux P. Neurogenic tumors of the stomach. Report of 18 cases and review of the literature. Rofo 1983;139:192-8.

9. Bees NR, Ng CS, Dicks-Mireaux C, Kiely EM. Gastric malignant schwannoma in a child. Br J Radiol 1997;70:952-5.

10. Hong HS, Ha HK, Won HJ, Byun JH, Shin YM, Kim AY, et al. Gastric schwannomas: radiological features with endoscopic and pathological correlation. Clin Radiol 2008;63:536-42.

11. Jung MK, Jeon SW, Cho CM, Tak WY, Kweon YO, Kim SK, et al. Gastric schwannomas: endosonographic characteristics. Abdom imaging 2008;33:388-90.

12. Lin CS, Hsu HS, Tsai CH, Li WY, Huang MH. Gastric schwannoma. J Chin Med Assoc 2004;67:583-6.

13. Snyder RA, Harris E, Hansen EN, Merchant NB, Parikh AA. Gastric schwannoma. Am Surg 2008;74:753-6.

14. Karabulut N, Martin DR, Yang M. Gastric schwannoma: MRI findings. Br J Radiol 2002;75:624-6.

15. Ahmed AR, Watanabe H, Aoki J, Shinozaki T, Takagishi K. Schwannoma of the extremities: the role of PET in preoperative planning. Eur J Nucl Med 2001;10:1541-51.

16. Hirai K, Umesaki N, Sumi T, Isiko O, Kanaoka Y, Ogita S, et al. Combined diagnostic imaging for retroperitoneal schwannoma. Oncol Rep 2001;8:773-5.

17. Toyama E, Nagai Y, Baba Y, Yoshida N, Hayashi N, Miyanari $\mathrm{N}$, et al. A case of thoracoscopically resected benign esophageal schwannoma with high uptake on FDG-PET. Esophagus 2008;5: 167-70.

18. Beaulieu S, Rubin B, Djang D, Conrad E, Turcotte E, Eary JF. Positron emission tomography of schwannomas: emphasizing its potential in preoperative planning. AJR Am J Roentgenol 2004;182:971-4.

19. Hou YY, Tan YS, Xu JF, Wang XN, Lu SH, Ji Y, et al. Schwannoma of the gastrointestinal tract: a clinicopathological, immunohistochemical and ultrastructural study of 33 cases. Histopathology 2006;48:536-45.

20. Kawabe J, Okumura T, Shakudo M, Koyama K, Wanibuchi H, Sakamoto $\mathrm{H}$, et al. Two cases of chronic tonsillitis studied by FDG-PET. Ann Nucl Med 1999;13:277-9. 